\title{
Intracardiac echocardiography-guided transseptal puncture for flouroless catheter ablation of left-sided tachycardias
}

\author{
David Žižek ${ }^{1}$, Bor Antolič ${ }^{1}$, Tine Prolič Kalinšek ${ }^{2}$, Jernej Štublar ${ }^{1}$, Nina Kajdič ${ }^{1}$, Matija \\ Jelenc $^{3}$, and Matevž Jan ${ }^{2}$ \\ ${ }^{1}$ University Medical Centre Ljubljana Division of Internal Medicine \\ ${ }^{2}$ University Medical Centre Ljubljana \\ ${ }^{3}$ University Medical Center Ljubljana
}

June 9, 2020

\begin{abstract}
Introduction Integration of intracardiac echocardiography (ICE) and 3D electroanatomic mapping (EAM) system allows transseptal punctures (TSP) without the use of fluoroscopy. Compared to fluoroscopy, ICE provides better visualization of the anatomy relevant to TSP and early recognition of complications. The aim was to evaluate efficacy and safety of entirely ICE- guided TSPs in patients who underwent fluoroless catheter ablation of left-sided tachycardias. Methods and results Consecutive 524 adult and pediatric patients referred to our institution from July 2014 to December 2019 were analyzed. Patients with cardiac implantable electronic devices (CIEDs) were also included. All procedures were performed with ICE-guided TSP combined with 3D EAM. Adverse events fol-lowing TSP and within 30 days of the procedure were analyzed. Altogether 949 TSPs (363 double punctures, $76.5 \%)$ were performed in 586 fluoroless ablation procedures: $451(77 \%)$ were ablation of atrial fibrillation or atypical flutter, 75 (12.8\%) of left-sided accessory pathway, $33(5.6 \%)$ of ventricu-lar tachycardia, and 27 (4.6\%) of focal atrial tachycardia. Forty-six $(7.8 \%)$ procedures were performed in pediatric population and 36 procedures $(6.1 \%)$ in patients with CIED. Only 2 TSPs were unsuc-cessful (2/949, 0.2\%). Overall procedural complication rate was 1.9\% (11/586 procedures). There was only 1 TSP related pericardial tamponade (2/949, 0.2\%). In CIED patients there was 1 lead dislo-cation following TSP. Conclusion Entirely ICE-guided TSPs for different left-sided tachycardias can be safely and effectively performed in adult and pediatric population without the use of fluoroscopy. However, caution is advised in CIED patients due to possible lead dislocation risk.
\end{abstract}

\section{Intracardiac echocardiography-guided transseptal puncture for flouroless catheter ablation of left-sided tachycardias}

Brief title: Fluoroless transseptal puncture

David Žižek, $\mathrm{MD}, \mathrm{PhD}^{1}$, Bor Antolič, MD, $\mathrm{PhD}^{1}$, Tine Prolič Kalinšek, $\mathrm{MD}^{2}$, Jernej Štublar, MSc ${ }^{1}$, Nina Kajdič, $\mathrm{MD}^{1}$, Matija Jelenc, $\mathrm{MD}, \mathrm{PhD}^{2}$, Matevž Jan, $\mathrm{MD}^{2}$

${ }^{1}$ Cardiology Department, University Medical Centre Ljubljana, Ljubljana, Slovenia

${ }^{2}$ Cardiovascular Surgery Department, University Medical Centre Ljubljana, Ljubljana, Slovenia

Correspondence: David Žižek, MD, PhD. Department of Cardiology, University Medical Centre Ljubljana, Zaloška 7, 1000 Ljubljana, Slovenia. Tel: + 386152285 34; fax: +386 152228 28; e-mail: david.zizek@kclj.si. ORCID: https://orcid.org/0000-0001-5029-3989

Conflict of interest: David Žižek received consulting/advisory board support from Medtronic and Boston Scientific. Bor Antolič and Matevž Jan received proctorship fees from Biosense Webster. All other authors 
have no relationships relevant to the contents of this paper to disclose.

Funding: the study did not receive any financial support.

\section{Structured Abstract}

\section{Introduction}

Integration of intracardiac echocardiography (ICE) and 3D electroanatomic mapping (EAM) system allows transseptal punctures (TSP) without the use of fluoroscopy. Compared to fluoroscopy, ICE provides better visualization of the anatomy relevant to TSP and early recognition of complications. The aim was to evaluate efficacy and safety of entirely ICE- guided TSPs in patients who underwent fluoroless catheter ablation of left-sided tachycardias.

\section{Methods and results}

Consecutive 524 adult and pediatric patients referred to our institution from July 2014 to December 2019 were analyzed. Patients with cardiac implantable electronic devices (CIEDs) were also included. All procedures were performed with ICE-guided TSP combined with 3D EAM. Adverse events following TSP and within 30 days of the procedure were analyzed. Altogether 949 TSPs (363 double punctures, $76.5 \%$ ) were performed in 586 fluoroless ablation procedures: $451(77 \%)$ were ablation of atrial fibrillation or atypical flutter, 75 $(12.8 \%)$ of left-sided accessory pathway, $33(5.6 \%)$ of ventricular tachycardia, and $27(4.6 \%)$ of focal atrial tachycardia. Forty-six (7.8\%) procedures were performed in pediatric population and 36 procedures $(6.1 \%)$ in patients with CIED. Only 2 TSPs were unsuccessful $(2 / 949,0.2 \%)$. Overall procedural complication rate was $1.9 \%$ (11/586 procedures). There was only 1 TSP related pericardial tamponade $(2 / 949,0.2 \%)$. In CIED patients there was 1 lead dislocation following TSP.

\section{Conclusion}

Entirely ICE-guided TSPs for different left-sided tachycardias can be safely and effectively performed in adult and pediatric population without the use of fluoroscopy. However, caution is advised in CIED patients due to possible lead dislocation risk.

Key words: transseptal puncture, intracardiac echocardiography, 3-dimensional systems, fluoroless, leftsided tachycardias

\section{Unstructured Abstract}

Compared to fluoroscopy, intracardiac echocardiography (ICE) provides better anatomical visualization and early recognition of complications related to transseptal puncture (TSP). A total of 949 ICE-guided TSPs (586 fluoroless left-sided arrhythmia procedures) performed in adult and pediatric patients were retrospectively analyzed. With a $99.8 \%$ success rate and only 1 transseptal access related pericardial tamponade $(0.2 \%)$, entirely ICE-guided TSP can be successfully and safely performed in fluoroless CA of left-sided arrhythmias. As 1 lead dislocation was recorded following TSP caution is advised in patients with intracardiac electronic devices.

\section{Abbreviations}

$3 \mathrm{D}=$ three-dimensional

$\mathrm{AF}=$ atrial fibrillation

$\mathrm{AP}=$ accessory pathway

$\mathrm{AT}=$ atrial tachycardia

AVNRT $=$ atrioventricular nodal reentry tachycardia

$\mathrm{CA}=$ catheter ablation

CIED $=$ cardiac implantable electronic device 


$$
\begin{aligned}
& \mathrm{EAM}=\text { electro-anatomic mapping } \\
& \mathrm{IAS}=\text { interatrial septum } \\
& \mathrm{ICE}=\text { intracardiac echocardiography } \\
& \mathrm{PTA}=\text { percutaneous angioplasty } \\
& \mathrm{RF}=\text { radio-frequency } \\
& \mathrm{SD}=\text { standard deviation } \\
& \mathrm{SVT}=\text { supra-ventricular tachycardia } \\
& \mathrm{TEE}=\text { transesophageal echocardiography } \\
& \mathrm{TSP}=\text { transseptal puncture } \\
& \mathrm{VT}=\text { ventricular tachycardia }
\end{aligned}
$$

\section{Introduction}

The transseptal puncture (TSP) is a routine procedure in electrophysiology to perform catheter ablation (CA) of atrial fibrillation (AF) and other left-sided arrhythmias including ventricular tachycardias (VT) (1).

Although fluoroscopic guidance to perform TSP is still the gold standard in clinical practice $(2,3)$, ultrasoundbased techniques including transesophageal echocardiography (TEE) and intracardiac echocardiography (ICE) have been adopted to provide additional imaging of different anatomical structures relevant to TSP. Utilization of both imaging techniques could shorten fluoroscopy exposure, increase safety and success rate of electrophysiological procedures, especially in challenging cases (4-6). Furthermore, improvement of image quality and integration with three-dimensional 3D electro-anatomic mapping (EAM) systems enabled ICE to become an invaluable tool for safe and effective guidance of catheters and needle direction during TSP without the use of fluoroscopy (7). While the fundamental technique of performing ICE-based TSP in flouroless approach remains the same, available data to support routine use of this technique is relatively scarce. In addition, there are some considerations regarding safety and efficacy, especially in the pediatric population and in patients with cardiac implantable electronic devices (CIEDs) $(8,9)$.

The purpose of this single-center retrospective study was to evaluate efficacy and safety of consecutive entirely ICE-guided TSPs in patients undergoing fluoroless CA for different left-sided tachycardias. Pediatric population and patients with CIEDs were also included in the analysis.

\section{Methods}

The study complies with the Declaration of Helsinki. The study and fluoroless CA procedures were approved by The National Medical Ethics Committee (Komisija za medicinsko etiko Republike Slovenije) and the institutional review board. All patients (or their parents in case of pediatric patients) gave written informed consent before ablation procedures were performed.

\section{Patient population}

In this retrospective study we included 524 consecutive adult and pediatric patients who were referred to our institution from July 2014 to December 2019 requiring TSP for CA of left-sided arrhythmias. Fluoroless approach in our center was gradually implemented for different CA procedures. Consequently, patients with left-sided tachycardias were included in this retrospective study at different points in time with consecutive TSPs thereafter: left-sided supra-ventricular tachycardias (SVTs) since July 2014, AF since March 2015 and left-sided VT since March 2017.

Left sided focal atrial tachycardia (AT), left-sided accessory pathway (AP) mediated tachycardia, atrioventricular nodal reentry tachycardia (AVNRT), or left-sided VT typically received single transseptal access. 
Patients with AF and left-sided atypical atrial flutters typically received double TSPs, except patients undergoing hybrid (combined surgical and catheter ablation) procedure for AF which received single transseptal access. Typically, the radio-frequency $(\mathrm{RF})$ needle technique was used in difficult TSP cases. Pediatric patients and patients with CIEDs were also included. Each CIED was tested for proper function before and after the procedure to ensure no change in pacing and sensing parameters occurred. Lead visualization during procedures was entirely performed with ICE without fluoroscopy.

\section{Procedural workflow}

Procedures were generally performed in conscious sedation, except in pediatric patients ( $<14$ years) and patients undergoing hybrid procedure for AF who received general anesthesia. Bilateral femoral vein punctures were performed to access the heart. All procedures were performed with 3D EAM system (NavX; Abbott, IL, USA or Carto®; Biosense Webster, CA, USA) and ICE (Acunav; Biosense Webster, CA, USA). All parts of the procedures were performed completely without the use of fluoroscopy, including mapping and ablation.

All patients received a full therapeutic dose of heparin before TSP and majority of AF patients had an uninterrupted anticoagulation strategy. Activated clotting time was regularly checked after having achieved transseptal access with a target of at least 300 seconds. The SR0 or SL1 long sheaths with dilators (Abbott, IL, USA) and HeartSpan(r) transseptal needle (71cm, $50 \%$ curve, 21 Gauge; MeritMedical, Utah, USA) were typically used for TSP. In case of the "RF needle" technique a full metal BRK transseptal needle $(71 \mathrm{~cm}$, $50 \%$ curve, 18 Gauge; Abbott, IL, USA) was used.

\section{Intracardiac echocardiography-guided transseptal puncture}

First, the ICE probe was advanced from the femoral vein into the right atrium. ICE probe was then further advanced into the right ventricle and the right ventricular outflow tract to visualize left atrial appendage and exclude the presence of possible thrombotic masses. Subsequent entirely ICE-guided workflow for achieving transseptal access is shown in Figure 1.

ICE was used to visualize the position of the guide wire and later the position and the course of the long sheath / dilator / transseptal needle assembly during the pull-down maneuver. Amid this maneuver the aortic root, pulmonary veins and the interatrial septum were used as landmarks which were repeatedly visualized with a slight clockwise or counterclockwise rotation of the ICE probe. Postero-inferior part of the oval fossa just opposite to the posterior aspect of the left pulmonary vein antrum was the target for landing the dilator tip in preparation for the TSP. ICE was also used to confirm the presence, orientation and exact location of the transseptal needle tip, the dilator tip, the long sheath tip and the guide wire in the left atrial cavity during different stages of transseptal puncture.

In addition, ICE was used to monitor the pericardial space during the procedure in a case of chest discomfort or arterial pressure drop. This was achieved with advancing the ICE probe into the right ventricle and rotating it clockwise until left ventricular cavity and surrounding pericardial space were visualized.

\section{Radio-frequency needle technique}

In difficult cases with initially unsuccessful TSP due to stiff or thickened IAS the RF needle technique was utilized. The main advantage of this technique is that significant mechanical force is not needed for successful and safe TSP (10). In short, after the long sheath / dilator / transseptal needle assembly was positioned at the optimal location on the IAS (panel D, Figure 1), the transseptal needle was pushed against the oval fossa. During consistent pressure of the needle and under uninterrupted ICE guidance the RF energy was applied to the shaft of the metal needle with the ablation catheter. Due to the expected rise of the impedance the high impedance cutoff on the RF generator was set to the maximum value. The RF energy of $20 \mathrm{~W}$ was generally applied for $5-10$ seconds until the tip of the needle was visualized in the LA cavity (panel E, Figure 1). The subsequent steps of TSP were as previously described in Figure 1.

\section{Transeptal puncture in patients with intracardiac devices}


When TSP was performed in a presence of a CIED its interrogation with testing lead sensing, pacing threshold and impedance was performed before and after the procedure. Also, lead position was repeatedly visualized with ICE during the pull-down maneuver of the long sheath/dilator/needle assembly to avoid possible entanglement and lead dislocation. X-ray fluoroscopy was not used for TSP in patients with CIEDs.

\section{Adverse events}

Major adverse events were defined as those causing termination of the procedure, prolonged hospital stay, long-term disability, requiring surgical intervention, CIED lead repositioning or bleeding requiring transfusion. In general, we focused on adverse events that could be directly attributed to TSP. Pericardial effusions occurring after completion of ablation procedures were not attributed to TSP. Periprocedural adverse events were monitored within 30-days of the ablation procedure. Cardiac perforation was suspected when the symptoms such as substernal discomfort, shoulder discomfort, abdominal discomfort, nausea, or clinical signs of hemodynamic instability were detected and pericardial effusion was confirmed with ICE or transthoracic echocardiogram. Pericardial effusion that necessitated intervention was defined as cardiac tamponade.

\section{Statistical analysis}

The baseline characteristics were reported as mean and standard deviation (SD) for numeric measures and counts and percentages for categorical measures. Successful TSPs and complications were reported as percentages. Reporting of cases with unsuccessful TSP, use of RF needle technique and similar deviations were only descriptive since the number of events was low.

\section{Results}

Altogether 949 TSPs were performed during 586 procedures in 524 patients. Patient characteristics are shown in Table 1. The majority of procedures were performed for AF or atypical atrial flutter. However, TSPs in other left-sided arrhythmias were also assessed: VT (5.6\%), AT (4.6\%), AP mediated tachycardia and AVNRT with left sided location of the slow pathway (12.8\%). Procedural characteristics are shown in Table 2. 57 patients had two (46 for AF, 3 for atypical atrial flutter, 5 for AP mediated tachycardia, 2 for focal AT, 1 for VT) and 5 patients had three procedures (3 for AF, 1 for and 1 for AP mediated tachycardia). Double TSPs were performed in majority of procedures for AF or atypical atrial flutter $(350 / 451,78 \%)$ and in a minority of procedures for focal atrial tachycardia $(8 / 27,30 \%)$ and VT $(5 / 33,15 \%)$. There were no double TSPs in patients treated for AP mediated tachycardia and AVNRT.

Only few procedures $(41 / 586,7 \%)$ were performed under general anesthesia; in pediatric patients who were under 14 years of age (22 procedures) and in patients undergoing hybrid ablation for persistent atrial fibrillation (19 procedures).

Only two TSPs were unsuccessful $(2 / 949,0.2 \%)$, both in patients with AF. In one patient with prior surgical removal of myxoma the operator was unable to puncture through the stiff IAS. The transseptal access was later successfully performed using RF needle technique. In the second unsuccessful TSP the presence of inferior vena cava filter precluded the femoral venous access. Subsequently right transjugular access was attempted but the operator was unable to insert a steerable or non-steerable long sheath into the left atrial cavity due to acute angle between the superior vena cava (SVC) and the IAS. Two procedures in patients with $\mathrm{AF}$ were aborted due to a visible mobile structure on the wire/long sheath/dilator assembly $(2 / 586,0.3 \%)$. In all cases, mapping and ablation parts of the procedure after successful TSP were performed completely without the use of fluoroscopy.

\section{Transseptal puncture in pediatric patients}

Forty-six $(7.8 \%)$ procedures with single TSPs were performed in pediatric population. Of those, 41 procedures were performed for AP mediated tachycardia, 4 for focal AT and 1 for fascicular VT. Seven $(7 / 46,15 \%)$ were performed in patients weighing less than $30 \mathrm{~kg}$. The youngest patient was 4 years old and weighted 17 $\mathrm{kg}$. Mean age and weight of pediatric patients were $12.5+-4$ years and $51.8+-19.5 \mathrm{~kg}$, respectively.

\section{Transseptal puncture in patients with intracardiac devices}


Thirty-six procedures $(6.1 \%)$ with 51 TSPs were performed in the presence of CIED (28 TSPs in single chamber devices, 21 TSPs in dual chamber devices and 2 TSPs in cardiac resynchronization device). One patient (1/51 punctures, $2 \%$ ) with a dual chamber device had procedure related dislocation of the ventricular lead that occurred at the time of achieving single transseptal access for VT ablation. There were no other lead dislocations and no changes in lead parameters according to pre and post-procedural device interrogations.

\section{RF needle technique}

Four difficult single TSPs $(4 / 949,0.4 \%)$ were successfully performed with the RF needle technique: 2 punctures in consecutive procedures in a patient with prior surgery of atrial myxoma, 1 TSP in a patient after previous AF ablation procedure, and 1 puncture where initial ICE evaluation revealed hypertrophied IAS. In the patient after previous AF ablation procedure, the percutaneous angioplasty (PTA) balloon was used to dilate the septal puncture site which enabled left atrial access with the long sheath. This was the only case where 7 seconds of low dose fluoroscopy (2 frames per second) was used during PTA balloon positioning and inflation. There were no adverse events in patients where RF needle technique was used.

\section{Adverse events}

Overall procedural complication rate was $1.9 \%$ (11/586 procedures). We registered 6 pericardial effusions that occurred after the completion of CA procedures and resolved spontaneously, causing only prolonged hospital stay. These pericardial effusions could not be attributed to TSP. Also, there were two femoral artery pseudoaneurysms requiring surgical intervention and one transient phrenic nerve palsy causing disability during the six-month recovery period. Detailed information on adverse events is provided in Table 3.

Importantly, adverse event rate that could be unequivocally attributed to the transseptal access was only $0.3 \%$ (3/949). Of note, one transseptal access related adverse event was CIED ventricular lead dislocation. Lead repositioning was performed during the same hospitalization. There was only 1 pericardial tamponade requiring intervention recorded immediately after double TSP was performed (2/949, 0.2\%). Since this was a double transseptal puncture and it was not clear which of the punctures resulted in tamponade, both were registered as adverse events. The cause for tamponade in this case was probably inadvertent puncture of the opposite left atrial wall during advancement of the transseptal needle in the presence of a small left atrium and a floppy interatrial septum.

\section{Discussion}

Our single-center retrospective study showed high success rate and very low complication rate of entirely ICE-guided TSPs for fluoroless CA treatment of left-sided tachycardias. Safety was also maintained in the pediatric population and, to a lesser degree, in patients with CIEDs.

\section{Intracardiac echocardiography-guided transseptal puncture}

To facilitate TSP, ICE or TEE are used as additional imaging tools when difficult anatomy is encountered during fluoroscopy-guided TSP $(5,6)$. Although cost-effectiveness in routine practice compared to TEE is unclear, ICE has additional advantages of not requiring a second operator, general anesthesia and provides better acoustic window. Furthermore, integration of ICE with 3D EAM systems enabled CA procedures of various arrhythmias without the use of fluoroscopy $(7,11)$. There are several reasons to adopt radiationreducing strategies in CA procedures. The ALARA (as low as reasonably achievable) principle is based on the hypothesis that there is no threshold below which ionizing radiation is free from harmful effects for the operator and the patient (12). Furthermore, patient radiation risk from CA procedures may highly exceed radiation risk from common radiological procedures (13).

There is limited data regarding feasibility and safety of ICE-guided TSP in CA of left-sided arrhythmias without the use of fluoroscopy. Recently, Baykaner al. (9) demonstrated efficacy and safety of zero fluoroscopy TSPs in $646 \mathrm{AF}$ patients using multiple procedural approaches at 5 high-volume centers. Our results extend these finding with a more simplified fluoroless transseptal approach which was also used in a pediatric population, in patients with CIEDs, and in various left-sided arrhythmias not only AF. 


\section{Transseptal puncture in pediatric patients}

In our analysis, all 46 pediatric patients with left-sided AP mediated tachycardia had successful TSPs without any complications. Present findings are in line with Clark et al. (14) who presented transseptal procedures in 9 pediatric patients without the use of fluoroscopy. Compared to the latter study, we also included smaller children weighing less than $30 \mathrm{~kg}$ where ICE was particularly useful to control the needle direction and monitor the space between the bulging IAS and LA wall with very small maneuvering space.

\section{Transseptal puncture in patients with intracardiac devices}

Available data in the literature on fluoroless TSP in the presence of CIEDs is scarce. Fluoroless approach for transseptal access is usually avoided or fluoroscopy is used at least to some extent during the procedure (9). In our study, 51 TSPs in 36 CIED patients were entirely ICE-guided with good visualization of the leads. However, we recorded one dislocation of the ventricular lead during TSP in VT ablation procedure. Lead dislocation due to entanglement with catheter system was intraoperatively detected with ICE. Thus, in patients with CIED caution is advised when attempting entirely ICE-guided TSP. Brief use of fluoroscopy for CIED lead visualization during long sheath / dilator/ transseptal needle assembly and during different stages of the CA procedure is probably reasonable if entanglement is suspected.

\section{Difficult transseptal puncture}

Anticipation and definition of difficult TSP is challenging even with additional preprocedural cardiac imaging. Previous AF ablation procedures and prior surgical or percutaneous interventions in the IAS area increase the risk for difficult TSP $(5,15,16)$. Although punctures with the aid of the RF needle technique that could be considered difficult in our study were low, we experienced no safety or efficacy issues with fluoroless ICE-guided approach. There were two patients in whom difficult TSP could be anticipated: one had previous transseptal access for AF ablation and second had prior surgical procedure on IAS. On the other hand, we can speculate that direct visualization of soft tissue structures and anatomical variations with ICE provides better reassurance of optimal puncture site compared to fluoroscopy and reduces the number of potentially difficult TSPs. Consequently, difficult cases could be reduced to just cases with stiff or thickened IAS / oval fossa where RF needle technique can be utilized to increase safety and efficacy (10).

\section{Adverse events}

Overall procedural complication rate in our study was low (1.9\%) and adverse events unequivocally attributable to TSP were even lower. In the setting of uninterrupted anticoagulation, there are several mechanisms of cardiac perforation during CA procedure including mechanical trauma of thin LA walls, coronary sinus perforation and steam pops during RF energy delivery. However, with various technological improvements of ablation catheters to reduce perforation risk TSP seems to be the pivotal step in achieving CA procedure safety. We observed only one pericardial tamponade following double transseptal access for AF ablation procedure, which accounts for only $0.2 \%$ of all TSPs. Safety profile of our procedures is very similar to the recent publication on fluoroless ICE-guided TSPs by Baykaner et al. (9) that also reported very low pericardial tamponade $(0.2 \%)$ and overall complication rate $(0.7 \%)$. Studies with large number of predominantly fluoroscopy-guided CA procedures also report relatively low TSP-related complications rates of $0.8 \%$ and $0.7 \%$, respectively $(1,3)$. On the other hand, some "real life" registries record higher pericardial tamponade rates of $1.2 \%(2)$ and $1.3 \%$ (17), respectively. Different adverse event definitions, study populations, and CA techniques could have attributed to the discrepancies of the results. In addition, nonuse of ICE was recognized as one of the strongest independent predictors of cardiac perforation in recent analysis of 102,398 patients who underwent AF ablation (18). In line with our observations, the study acknowledges wider adoption of intraprocedural ICE use to increase safety of CA procedures.

\section{Clinical implications}

Transseptal access is the key step in radiation-reducing strategies for CA of left-sided arrhythmias. Our data suggests that an entirely ICE-guided TSP enables operators to achieve completely fluoroless ablation 
procedures without compromising safety and efficacy. Fluoroscopy-based studies report, albeit rarely, complications such as right atrial wall puncture, aortic root puncture and embolisms which were not detected in the present study $(1,2,18)$. Routine use of ICE does not only provide real time visualization of IAS anatomy relevant to TSP but also facilitates early recognition of potential procedural complications such as formation of thrombus, signs of impending steam pop or pericardial effusion. In our study we had two aborted cases due to early recognition of thrombus with ICE which in theory prevented an adverse event.

\section{Limitations}

The present study has some limitations. Retrospective design limits the strength of our findings. Collection of data at a single center may result in treatment bias, which could influence the outcomes. However, our study cohort was representative of patients who undergo CA procedures for left-sided arrhythmias (1-3, 9-11, 17-19). We did not specifically assess the potential learning curve for completely ICE-based TSP and CA ablation without the use of fluoroscopy. With limited number of procedures and low complication rate it seems that our study is underpowered to predict potential risk factors for pericardial tamponade and other TSP-related complications. However, it is reasonable to assume that the use of ICE prevented some of the potential adverse events. Our findings in pediatric population and in patients with CIEDs should be interpreted with caution since the numbers were low. Finally, potential anatomical variations seen with ICE were not recorded systematically, which limits our estimation of additional benefits of ICE imaging. Further clinical studies are needed to establish cost-effectiveness and clinical benefits of entirely ICE-guided TSPs.

\section{Conclusions}

Entirely ICE-guided TSPs for different left-sided tachycardias can be safely and effectively performed in adult and pediatric population without the use of fluoroscopy. However, caution is advised in patients with CIEDs due to possible risk of lead dislocation.

Author contributions: all authors (DŽ, BA, TPK, JŠ, NK, MJ and MJ) made substantial contribution to the design of the study, acquisition, analysis and interpretation of data. All authors also actively participated in the drafting the manuscript, its final revisions and corrections. All authors have read and approved the final version of the manuscript.

\section{References}

1. De Ponti R, Cappato R, Curnis A, Della Bella P, Padeletti L, Raviele A, Santini M, Salerno-Uriarte JA. Transseptal catheterization in the electrophysiology laboratory: data from a multicenter survey spanning 12 years. J Am Coll Cardiol 2006;47:1037-1042.

2. Steinbeck G, Sinner MF, Lutz M, Müller-Nurasyid M, Kääb S, Reinecke H. Incidence of complications related to catheter ablation of atrial fibrillation and atrial flutter: a nationwide in-hospital analysis of administrative data for Germany in 2014. Eur Heart J 2018;39:4020-4029.

3. Matoshvili Z, Bastani H, Bourke T, Braunschweig F, Drca N, Gudmundsson K, Insulander P, Jemtrén A, Kennebäck G, Saluveer O, Schwieler J, Tapanainen J, Wredlert C, Jensen-Urstad M. Safety of fluoroscopyguided transseptal approach for ablation of left-sided arrhythmias. Europace 2017;19:2023-2026.

4. Bayrak F, Chierchia GB, Namdar M, Yazaki Y, Sarkozy A, de Asmundis C, Muller-Burri SA, Rao J, Ricciardi D, Sorgente A, Brugada P. Added value of transoesophageal echocardiography during transseptal puncture performed by inexperienced operators. Europace 2012;14:661-665.

5. Enriquez A, Saenz LC, Rosso R, Silvestry FE, Callans D, Marchlinski FE, Garcia F. Use of intracardiac echocardiography in interventional cardiology: working with the anatomy rather than fighting it. Circulation 2018;137:2278-2294.

6. Goya M, Frame D, Gache L, Ichishima Y, Tayar DO, Goldstein L, Lee SHY. The use of intracardiac echocardiography catheters in endocardial ablation of cardiac arrhythmia: Meta-analysis of efficiency, effectiveness, and safety outcomes. J Cardiovasc Electrophysiol 2020;31:664-673. 
7. Reddy VY, Morales G, Ahmed H, Neuzil P, Dukkipati S, Kim S, Clemens J, D'Avila A. Catheter ablation of atrial fibrillation without the use of fluoroscopy. Heart Rhythm 2010;7:1644-1653.

8. Jan M, Žižek D, Rupar K, Mazić U, Kuhelj D, Lakič N, Geršak B. Fluoroless catheter ablation of various right and left sided supra-ventricular tachycardias in children and adolescents. Int J Cardiovasc Imaging 2016;32:1609-1616.

9. Baykaner T, Quadros KK, Thosani A, Yasmeh B, Mitra R, Liu E, Belden W, Liu Z, Costea A, Brodt CR, Zei PC. Safety and efficacy of zero fluoroscopy transseptal puncture with different approaches. Pacing Clin Electrophysiol 2020;43:12-18.

10. Winkle RA, Mead RH, Engel G, Patrawala RA. The use of a radiofrequency needle improves the safety and efficacy of transseptal puncture for atrial fibrillation ablation. Heart Rhythm 2011;8:1411-1415.

11. Sadek MM, Ramirez FD, Nery PB, Golian M, Redpath CJ, Nair GM, Birnie DH. Completely nonfluoroscopic catheter ablation of left atrial arrhythmias and ventricular tachycardia. J Cardiovasc Electrophysiol 2019;30:78-88.

12. Heidbuchel H, Wittkampf FH, Vano E, Ernst S, Schilling R, Picano E, Mont L, Jais P, de Bono J, Piorkowski C, Saad E, Femenia F. Practical ways to reduce radiation dose for patients and staff during device implantations and electrophysiological procedures. Europace 2014;16:946-964.

13. Perisinakis K, Damilakis J, Theocharopoulos N, Manios E, Vardas P, Gourtsoyiannis N. Accurate assessment of patient effective radiation dose and associated detriment risk from radiofrequency catheter ablation procedures. Circulation 2001;104:58-62.

14. Clark BC, Sumihara K, Berul CI, Moak JP. Off the pedal: Fluoroless transseptal puncture in pediatric supraventricular tachycardia ablation. Pacing Clin Electrophysiol 2017;40:1254-1259.

15. Sharma SP, Nalamasu R, Gopinathannair R, Vasamreddy C, Lakkireddy D. Transseptal Puncture: Devices, Techniques, and Considerations for Specific Interventions. Curr Cardiol Rep 2019;21:52.

16. Marcus GM, Ren X, Tseng ZH, Badhwar N, Lee BK, Lee RJ, Foster E, Olgin JE. Repeat transseptal catheterization after ablation for atrial fibrillation. J Cardiovasc Electrophysiol 2007;18:55-59.

17. Arbelo E, Brugada J, Hindricks G, Maggioni A, Tavazzi L, Vardas P, Anselme F, Inama G, Jais P, Kalarus Z, Kautzner J, Lewalter T, Mairesse G, Perez-Villacastin J, Riahi S, Taborsky M, Theodorakis G, Trines S; Atrial Fibrillation Ablation Pilot Study Investigators. ESC-EURObservational Research Programme: the Atrial Fibrillation Ablation Pilot Study, conducted by the European Heart Rhythm Association. Europace 2012;14:1094-1103.

18. Friedman DJ, Pokorney SD, Ghanem A, Marcello S, Kalsekar I,Yadalam S, Akar JG, Freeman JV, Goldstein L, Khanna R, Piccini JP. Predictors of cardiac perforation with catheter ablation of atrial fibrillation. J Am Coll Cardiol EP 2020 March 25 [E-pub ahead of print], https://doi.org/10.1016/j.jacep.2020.01.011.

Figure 


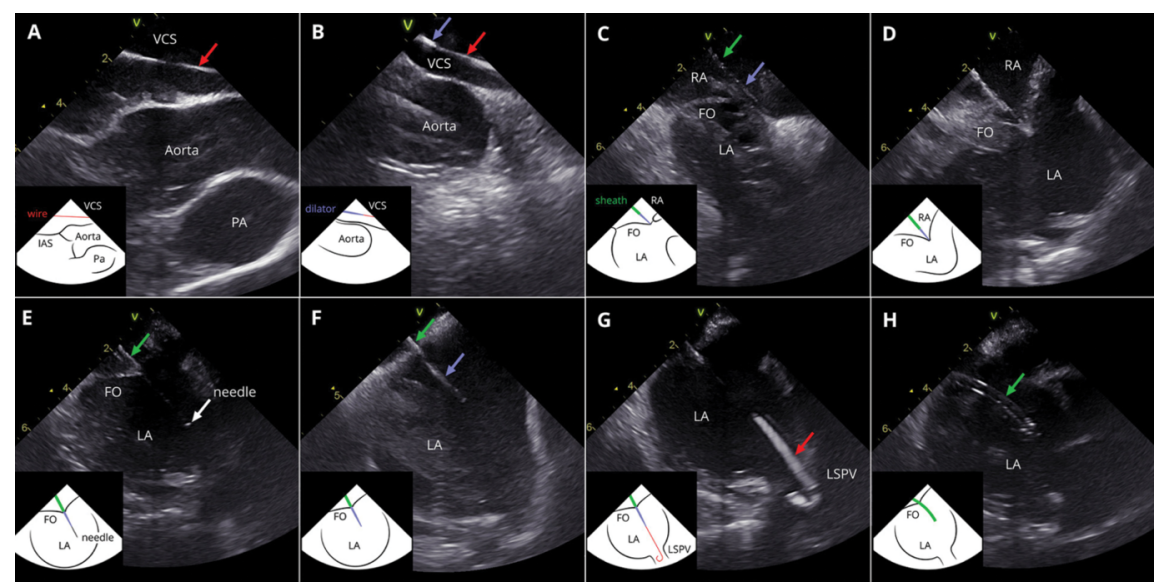

Figure 1. Intracardiac echocardiography-guided workflow for achieving transseptal access.

Images obtained with the ICE probe placed in the right atrium with added simplified graphic image in each panel for clarification. Panels A to $\mathrm{H}$ show consecutive steps in achieving transseptal access. The guide wire is marked with a red arrow in ICE images and represented with the red line in graphic images, the tip of the dilator is marked with a purple arrow in ICE images and represented with a purple line in graphic images, the tip of the transseptal needle is marked with a white arrow ICE images and represented with a black line in graphic images, the tip of the long sheath is marked a green arrow in ICE images and represented with a bolded green line in graphic images. VCS superior vena cava, PA pulmonary artery, RA right atrium, LA left atrium, FO oval fossa, LSPV left superior pulmonary vein.

A The guide wire is inserted into the VCS. B The long sheath with the dilator inserted is advanced into the VCS over the guide wire. C The guide wire is replaced with the transseptal needle and the long sheath / dilator / transseptal needle assembly is withdrawn toward the interatrial septum. D The tip of the dilator falls on the oval fossa and its "tenting" can be seen. E The transseptal needle is pushed through the dilator and advanced into the LA cavity together with the tip of the dilator. $\mathbf{F}$ The transseptal needle is withdrawn and the tip of the dilator remains in the LA cavity. G The guide wire is advanced through the dilator into the LSPV. H The long sheath and the dilator are advanced over the guide wire into the LA cavity. The guide wire and the dilator are withdrawn with the tip of the long sheath remaining in the LA cavity.

\section{Tables}

Table 1. Baseline patient characteristics.

\begin{tabular}{ll}
\hline Baseline characteristics & Overall $(\mathbf{n = 5 2 4})$ \\
\hline Age (Years) & $55.8 \pm 17.6$ \\
Male (\%) & $377(72)$ \\
Body Mass Index $\left(\mathrm{kg} / \mathrm{m}^{2}\right)$ & $27.8 \pm 5$ \\
Cardiovascular risk factors & \\
Arterial hypertension (\%) & $242(46)$ \\
Diabetes (\%) & $36(6.8)$ \\
Heart failure (\%) & $73(14)$ \\
Previous stroke / TIA (\%) & $10(1.9)$ \\
Vascular disease (\%) & $45(8.6)$ \\
$\mathrm{CHA}_{2} \mathrm{DS}_{2}$ VASc score & $1.4 \pm 1$ \\
\hline
\end{tabular}

TIA - transient ischemic attack; $\mathrm{CHA}_{2} \mathrm{DS}_{2} \mathrm{VASc}$ - stroke and thromboembolic risk score $(\mathrm{C}$ congestive heart 
failure, $\mathrm{H}$ hypertension, A age, D diabetes, $\mathrm{S}$ stroke, V vascular disease, A age 65-74 S sex category - female)

Table 2. Procedural characteristics.

\begin{tabular}{ll}
\hline Procedures & Overall $(\mathbf{n = 5 8 6})$ \\
\hline Mean duration (min) & $161 \pm 64$ \\
Procedures in general anesthesia (\%) & $41(7)$ \\
Procedure type & \\
AF and atypical flutter (\%) & $451(77)$ \\
Hybrid procedures for AF (\%) & $20(3.4)$ \\
Procedures for VT (\%) & $33(5.6)$ \\
Procedures for focal AT (\%) & $27(4.6)$ \\
Procedures for AP mediated tachycardia (\%) & $74(13)$ \\
Procedures for AVNRT (\%) & $1(0.2)$ \\
Transseptal punctures & Overall (n=949) \\
Double punctures (\%) & $726(76.5)$ \\
Presence of CIED (\%) & $51(5.4)$ \\
Pediatric patients (\%) & $46(4.8)$ \\
Pediatric patients $<30 \mathrm{~kg}(\%)$ & $7(0.7)$ \\
RF needle technique (\%) & $4(0.4)$ \\
Unsuccessful (\%) & $2(0.2)$ \\
\hline
\end{tabular}

AF - atrial fibrillation; VT - ventricular tachycardia; AT - atrial tachycardia; AP - accessory pathway; CIEDs - cardiac implantable electronic devices

Table 3. Procedural and transseptal access-related complications.

\begin{tabular}{lll}
\hline & Ablation procedure $(\mathbf{n = 5 8 6})$ & Transseptal access $(\mathbf{n}=\mathbf{9 4 9})$ \\
\hline Stroke / transient ischemic attack (\%) & 0 & 0 \\
Pericardial effusion (\%) & $6(1.2)$ & 0 \\
Pericardial tamponade (\%) & $1(0.2)$ & $2(0.2)$ \\
PSA surgery / intervention (\%) & $2(0.3)$ & 0 \\
Esophageal injury (\%) & 0 & 0 \\
Transient phrenic palsy (\%) & $1(0.2)$ & 0 \\
CIED lead dislocation (\%) & $1 / 36$ procedures $(2.7)$ & $1 / 51$ punctures $(2)$ \\
Total (\%) & $11(1.9)$ & $3(0.3)$ \\
\hline
\end{tabular}

PSA - pseudoaneurysm; CIEDs - cardiac implantable electronic devices 